\title{
Data Fusion in Wireless Sensor Networks using Fuzzy Systems
}

\author{
Awat Mandeh \\ Research Scholar \\ Department of Computer \\ Engineering, Sanandaj Branch, \\ Islamic Azad University, \\ Sanandaj, Iran
}

\author{
Keyhan Khamforoosh \\ Assistant Professor \\ Department of Computer \\ Engineering, Sanandaj Branch, \\ Islamic Azad University, \\ Sanandaj, Iran
}

\author{
Vafa Maihami \\ Facility Member \\ Department of Computer \\ Engineering, Sanandaj Branch, \\ Islamic Azad University, \\ Sanandaj, Iran
}

\begin{abstract}
Since battery is the source of energy for sensors, one of the important issues in wireless sensor networks is the energy and network lifetime. A method to reduce energy consumption and, as a result, increase the network lifetime is the fusion of data collected from the sensors in the covered environment before transmission to wireless sensor network. Data fusion in sensors is defined as the process in which the data received from multiple sources are integrated in order to achieve better perceived information with respect to only one source. In this paper, a new method is proposed for data fusion in network sensors using fuzzy systems. In the proposed method, by integrating the input data into each sensor, each of which had three inputs, the similarity percent of the data in sensors was obtained in order to identify the size of data (packets) to be sent. Simulation results on the proposed method verified the efficiency of the proposed method in terms of energy consumption in the network.
\end{abstract}

\section{Keywords}

Data fusion, Wireless sensor network, Fuzzy Systems, Reduce the energy consumption.

\section{INTRODUCTION}

Wireless sensor networks are the new technology in monitoring environments and distributed processing [1]. These networks consist of many small and inexpensive sensor nodes which are distributed throughout the environment. The application of these networks includes many fields such as military, industrial, monitoring the environment, household, medicine, agriculture, etc. In most of its applications, wireless sensor networks should have the ability to work autonomously without any human intervention within large environments $[14,15]$.One of the important challenges in wireless sensor networks is the energy and network lifetime. A method to reduce energy consumption and, as a result, increase the network lifetime is to integrate the data collected from the sensors that cover the environment before the data transmission through the network. Data fusion in sensors is defined as the process in which the data received from multiple sources are integrated in order to achieve better perceived information with respect to only one source [2].

In wireless sensor networks, using the data fusion at different levels, it is possible to reduce the data traffic load and improve the performance; also, a particular inference is obtained at each step and, finally, it is possible to get closer to the actual inference by integrating the results in each step [6]. Nonetheless, researchers are looking for solutions to improve the hardware and software technology by benchmarking from the combined capabilities of humans and animals in relation to natural data and to simulate new sensors by discovering some facts that can improve data fusion for providing more accurate inferences [2], [7].

In this paper, a new method is proposed for data fusion in network sensors using fuzzy systems. In the proposed method, first, three inputs, namely the neighbors, packets, and distance, were allocated to each sensor in the network. Next, a value or similarity in percentage was obtained by integrating the mentioned input data. Based on the obtained similarity percent, the number of transmitted data (packets) to be reduced, removed, or remained unchanged was found, which finally provided the network with more useful lifetime.

The rest of this paper is organized as follows. In Section 2, the fuzzy system is briefly explained. In Section 3, the designed system is presented in detail and the necessary figures and inferences are expressed. In Section 4, the system simulation is presented and the conclusion is provided in the final section.

\section{FUZZY SYSTEMS}

Different computational intelligence algorithms have been used for data fusion in wireless sensor networks [9-12]. One of the computational intelligence algorithms is fuzzy logic or fuzzy system algorithm. Many conventional methods in various sciences are not able to properly support a high volume of quantitative and qualitative information [8]. On the other hand, most of the information is associated with ambiguity and uncertainty. The fuzzy logic has provided a suitable mathematical formulation to these ambiguities and prepared the context for decision making and reasoning about them. Generally, nature and the environment are associated with complexity, dynamism, and uncertainty. The fuzzy logic can be effective in working with ambiguous data and complex models due to having simple and flexible concepts [3]. It is something more than precise and machine logic and works like human logic without requiring accurate data [4]. Classic logic represents everything in a crisp form as an absolute $(0$, $1)$; in contrast, the fuzzy logic always shows these values between zero and one. For example, if black and white are respectively considered zero and one, then, gray will be a number close to zero. Fuzzy logic was invented for the first time in 1965 by Dr. Lotfizadeh. Then, it was used in various academic and industrial fields of the fuzzy logic [13] [14]. In the proposed algorithm, fuzzification and defuzzification systems have the highest application. The main components of fuzzy systems are three parts including fuzzification, inference rules, and defuzzification (Figure 1). Crisp input data are fuzzified and, then, the necessary inference is taken according 
to the rules and membership functions that are defined in the system for the inputs. In the next step, the considered fuzzy outputs are produced and, finally, transformed into the crisp form which is acceptable by industrial machines. The main purpose of a fuzzy system is to archive a set of input and output connections for describing a process [5].

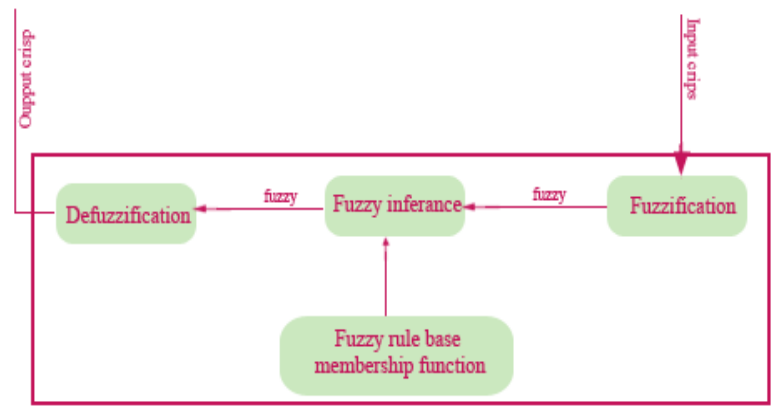

Fig 1: Fuzzy system components

Many methods can be used to show membership function. Equations 1 and 2 present two triangular and trapezoidal methods, which are used in most of the fuzzy systems owing to their higher clarity and greater simplicity.

Triangular membership function is as follows:

$A=\left\{\begin{array}{lr}0, & x \leq a \\ \frac{x-a}{b-a}, & x \in(a, b) \\ \frac{c-x}{c-b}, & x \in(b, c) \\ 0, & x \geq c\end{array}\right.$

And trapezoidal membership function is written as:

$A=\left\{\begin{array}{lr}0, & x \leq a \\ \frac{x-a}{b-a}, & x \in(a, b) \\ 1, & x \in(b, c) \\ \frac{d-x}{d-c}, & x \in(c, d)\end{array}\right.$

\section{THE PROPOSED ALGORITHM}

The methodology of the proposed algorithm has been built based on similarity. As seen in Figure 2, three inputs were allocated to the considered sensor for each second and each sensor; the input values were fuzzified in the fuzzification section. Based on the membership function of the inputs as defined in the database and a number of rules defined in the rules database, the necessary inference was made on the input data and, according to the interferences, it was found whether the similarity percent was high or low. The results were then defuzzified. After determining the percentages of similarity, the test step started, in which if the obtained similarity percent were high, some of the transmitted data (packets) would be reduced or eliminated; but, if the similarity percent were low, the transmitted data (packets) would be sent without the slightest change. This method not only increased the rate of data transmission, but also reduced the battery energy consumption of sensors, thus increasing the network's useful lifetime.

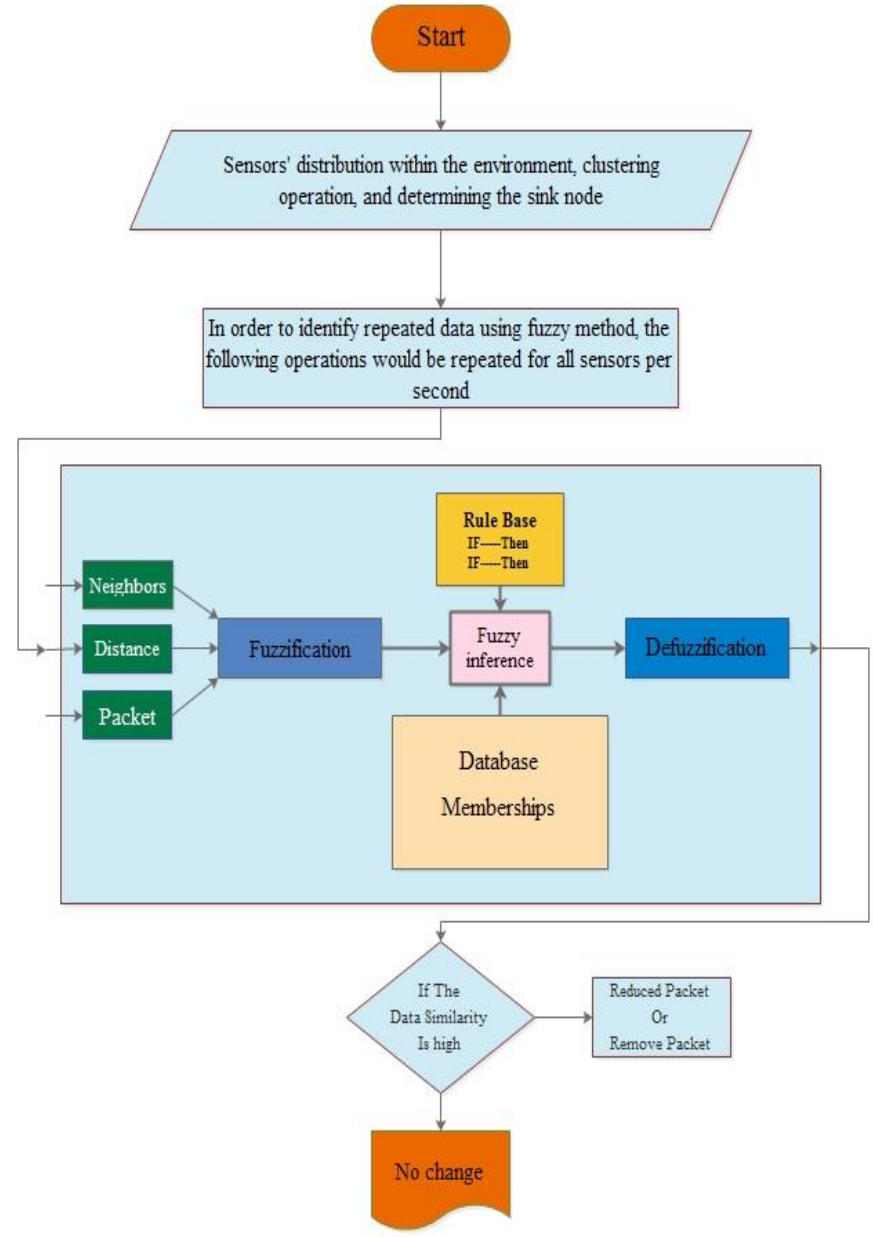

Fig 2: Flowchart of the proposed algorithm

\subsection{Describe the proposed algorithm}

Fuzzy algorithm inputs:

- Neighbors

- Distance

- $\quad$ Packet

Architecture of the proposed model is shown in Figure 3; but, the defuzzification section is not demonstrated.

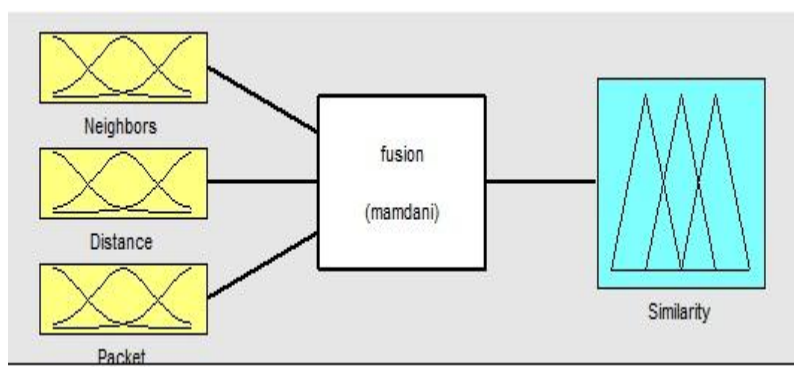

Fig 3: Model proposed architecture

\subsection{Definition of input and output membership functions}

To conduct a fuzzy process on the data collected by the sensors, first, the fuzzification process was performed on the inputs. For this purpose, all the input parameters of the system used low, medium, and high linguistic variables, while five variables, namely very low, low, medium, high, and very high, were used for output parameters. The fuzzy linguistic 
variables of the system inputs and outputs are shown in Table 1.

Table 1. Fuzzy linguistic variables for the fuzzification of the system parameters

\begin{tabular}{|c|c|c|}
\hline Parameters & Type & $\begin{array}{c}\text { Transformation into the fuzzy } \\
\text { language }\end{array}$ \\
\hline Neighbors & Input & Low-Normal-High \\
\hline Distance & Input & Low-Normal-High \\
\hline Packet & Input & Low-Normal-High \\
\hline Similarity & Output & $\begin{array}{c}\text { Very Low-Low-Normal-High- } \\
\text { Very High }\end{array}$ \\
\hline
\end{tabular}

Fig 4: Input fuzzy set, "Neighbors"

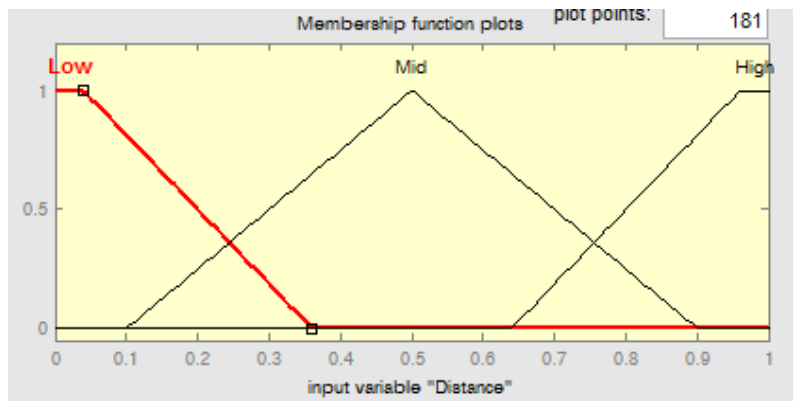

Fig 5: Input fuzzy set, "Distance"

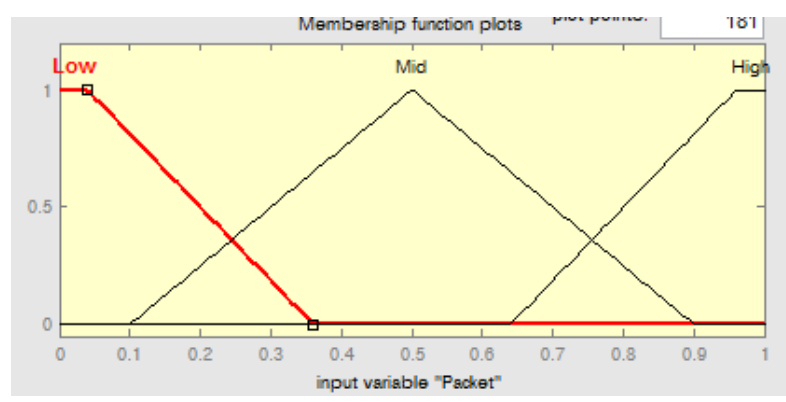

Fig 6: Input fuzzy set, "Packet"

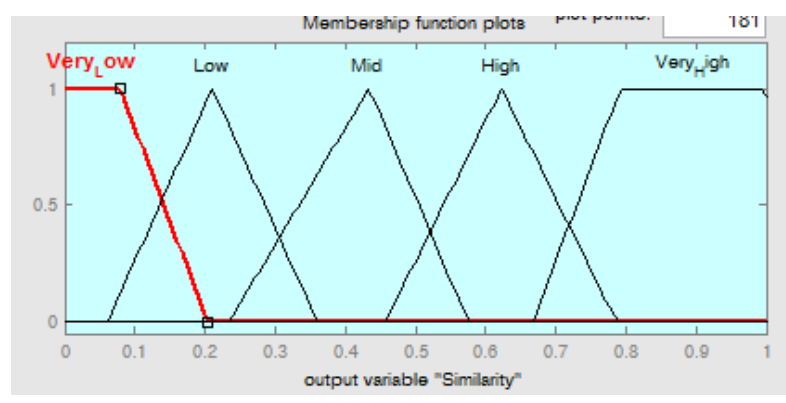

Fig 7: Output fuzzy set, "Similarity"
Figures 4, 5, and 6 show the input membership functions, while (Figure 7) presents the output membership function of the fuzzy system. Size of these functions is written arbitrarily and based on the view about the system without any special technique

\subsection{Developing the rule base}

To conduct inference on the inputs, in addition to defining the membership functions, the rule base is also developed. These rules are identified and written based on the environmental conditions of the sensors and the existing methodology and are generally determined arbitrarily in a conceptual fashion without any special condition. In the proposed algorithm, 27 rules were considered based on similarity conditions, because the written proposed algorithm had three inputs, each of which had three membership functions. For this reason, $27\left(3^{3}\right)$ conditions were considered. Table 2 shows these rules which were implemented in MATLAB environment. For example, in the first rule, if all the inputs were low, the similarity was also low. Four rules of the totally defined 27 rules are as follows.

Table 2. Fuzzy rule base

\begin{tabular}{|c|c|c|c|c|}
\hline Row & Packet & Distance & Neighbors & Similarity \\
\hline 1 & Low & Low & Low & Low \\
\hline 2 & Mid & Low & Low & Low \\
\hline$\cdot$ & $\cdot$ & $\cdot$ & $\cdot$ & $\cdot$ \\
$\cdot$ & $\cdot$ & $\cdot$ & $\cdot$ & $\cdot$ \\
\hline 28 & Mid & High & High & High \\
\hline 29 & High & High & High & High \\
\hline
\end{tabular}

Definition of the rule base and the connection of these rules are shown in the form of tables. In Figures 8, 9, and 10, the connection between these rules is shown in the surface mode.

The connection between the inputs is pairwise.

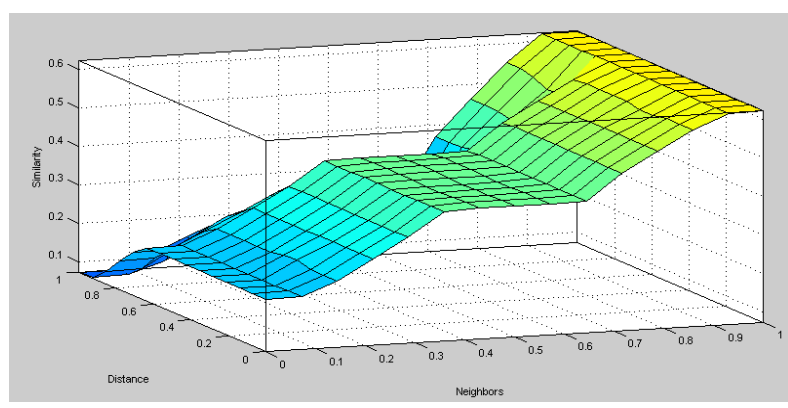

Fig 8: Surface view of similarity percentage- With respect to "neighbors" and "distance"

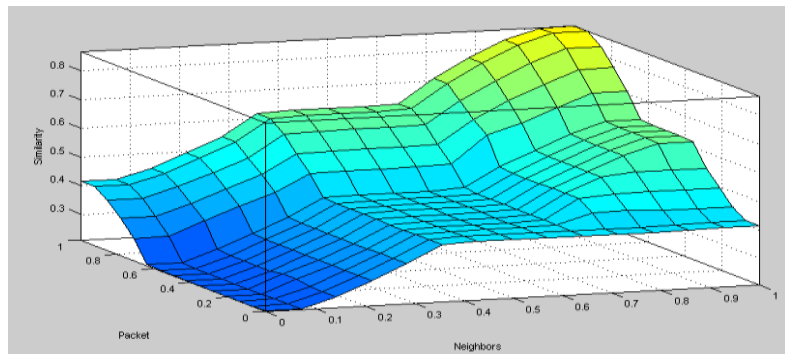

Fig 9: Surface view of similarity percentage- With respect to "neighbors" and "packet" 


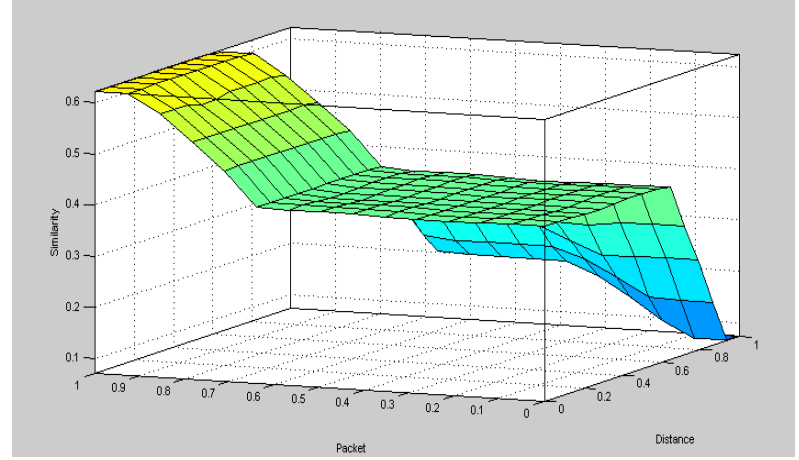

Fig 10: Surface view of similarity percentage- With respect to "packet" and "distance"

\section{SIMULATION RESULTS}

\subsection{SIMULATION AND DISCUSSION}

An environment which is going to be covered by a sensor network was assumed. The number of sensors was $N$ where $N=\{1,2, . . n\}$. Each sensor was randomly distributed throughout the environment. Also, there was a cluster head in each region which had a fixed position at the center of the region, where all the sensor nodes of the same region could directly communicate with it. There was a central node, also called sink, which was located in the environment. Figures 11 and 12 show the examples of this scenario along with arrangement of sensors and sink. The sensors were not connected directly to the sink and could communicate with the sink or base station only via cluster heads and relay nodes. This scenario of wireless sensor networks is known as two-tire wireless sensor networks.

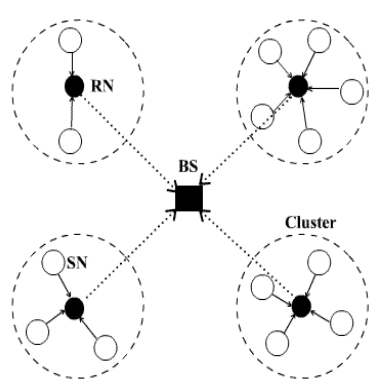

(a)

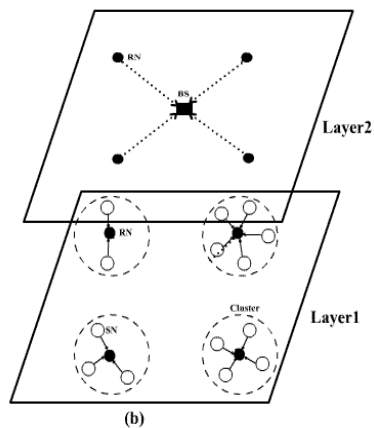

Fig 11: An example of arrangement of sensors and sink

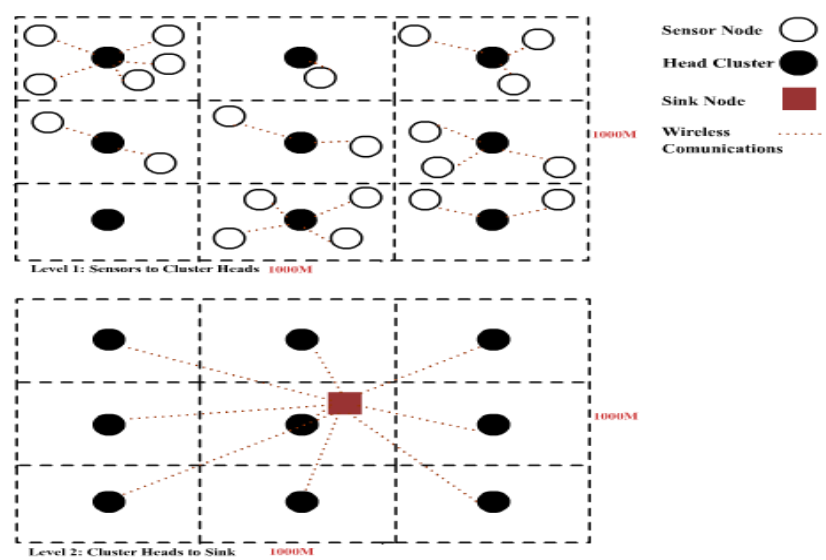

Fig 12: An example of arrangement of sensors and sink
The range of all sensors including the sink was assumed to be $400 \mathrm{~m}$. All the sensors were connected directly to the cluster head and could transmit their data to it; but, no sensor was directly connected to the sink and only the cluster heads were connected to the sink at the second level. All the cluster heads were also directly connected to the sink.

According to the proposed environment scenario, different results were obtained. The proposed algorithm was tested in three networks with different environments and the comparison was also made for two cases. The first case was the energy consumption and the second one was the number of transmitted packets where the results were compared in fuzzified and normal modes.

- Results based on an environment with 100 sensors

- Results based on an environment with 1000 sensors

- Results based on an environment with 10 sensors

Table 3. Simulation parameters of the network in a constant sink mode

\begin{tabular}{|c|c|}
\hline Parameters & Value \\
\hline Number of sensors in the environment & $10,100,1000$ \\
\hline Time simulation & $\begin{array}{c}\text { Duration }=30 \\
\text { Second }\end{array}$ \\
\hline Energy consumption per transmission & $75 \mathrm{MJ}$ \\
\hline Energy consumption per reception & $50 \mathrm{MJ}$ \\
\hline
\end{tabular}

The simulation parameters for all the three network cases are shown in Table 3. Only the number of the sensors is variable. In all the three network cases, the results were tested in a 30 sec interval. Energy consumption was considered 75 MJ per transmission in the network and $50 \mathrm{MJ}$ per received data. Energy consumption was achieved by adding these values with the obtained results

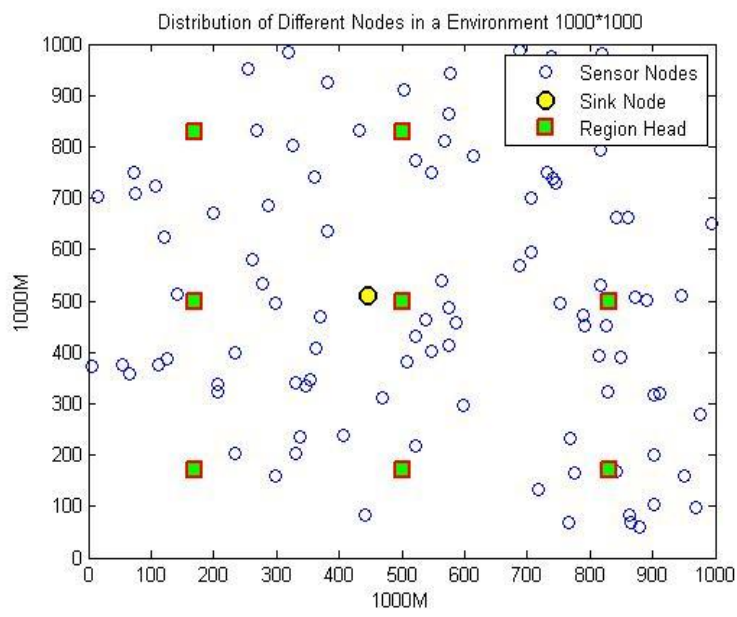

Fig 13: Random distribution of the sensors within the environment

The random distribution of the sensors in the environment is shown in Figure 13. 


\subsection{Results with 100 sensors}

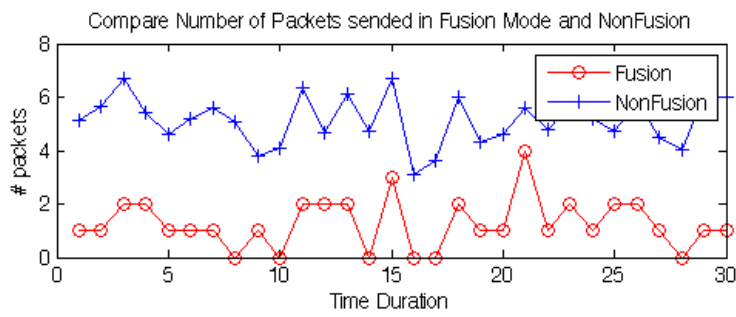

Fig 14: Results based on the consumed Jules by 100 sensors

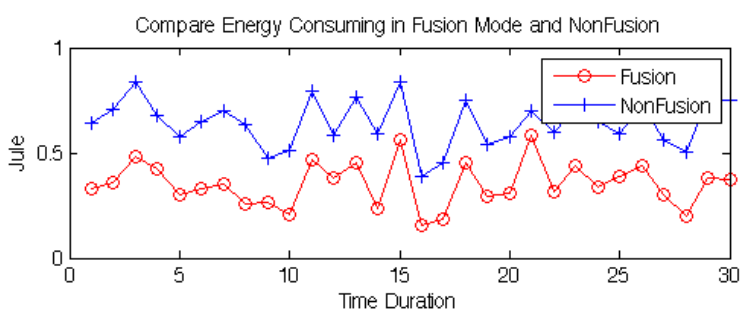

Fig 15: Results based on the number of packets transmitted by 100 sensors

In Figures 14 and 15, the evaluation results in fuzzification (red) and defuzzification (blue) modes are presented. As can be seen, when the fuzzification operation was not conducted and the network continued to work normally, the data were transmitted unchanged, without being reduced or removed. As a result, the energy consumption and number of transmitted packets were relatively high, thus reducing the network lifetime and also decreasing the rate of data transmission. It can be seen in the figures that, due to the similarity after the fuzzification was performed, the data were reduced or removed. Hence, the energy consumption and number of transmitted packets were decreased, thus causing a significant improvement in the network lifetime and rate of data transmission.

\subsection{Results with 1000 sensors}

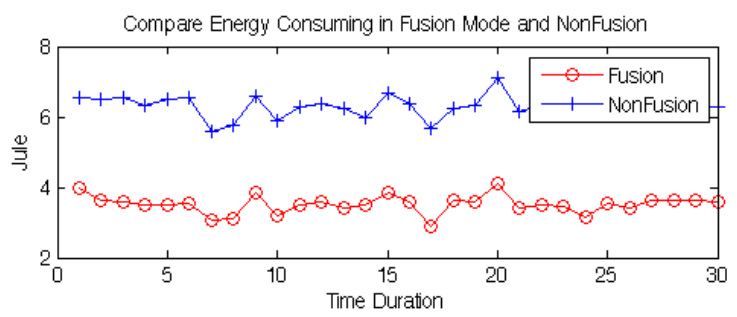

Fig 16: Results based on the consumed Jules by 1000 sensors

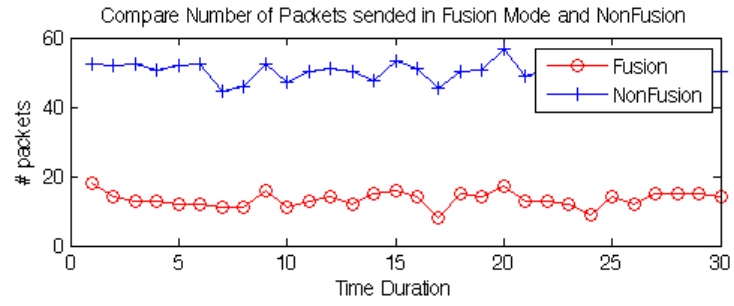

Fig 17: Results based on the number of packets transmitted by 1000 sensors

As can be seen in Figures 16 and 17, slight changes were made to the results compared with the pervious case, which still improved energy consumption and transmitted packets in the networks with a large number of sensors. Only small distance was created between fuzzification and defuzzification modes and the network energy consumption was reduced by greater values. The reason is that the number of sensors in the environment and, consequently, the number of sensors in the regions increased. According to what was explained in the proposed algorithm, a higher percentage of data was reduced or removed due to the increase in the similarity percent, since the number of sensors in the regions increased. By comparing the fuzzification mode with the defuzzification mode, it was concluded that increase in the number of sensors had a significant impact on the reduction of energy consumption and, as a result, enhancement of the network's useful lifetime, which would contribute to achieving better results.

\subsection{Results with 10 sensors}

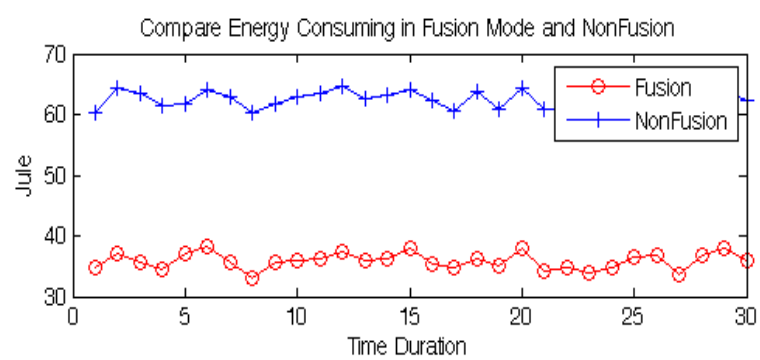

Fig 18: Results based on the consumed Jules by 10 sensors

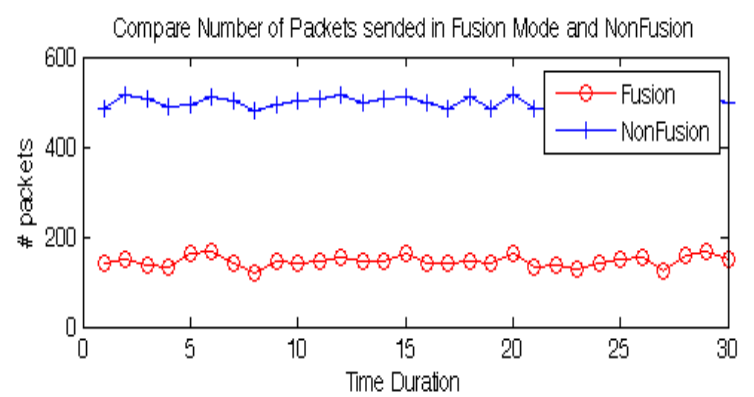

Fig 19: Results based on the number of packets transmitted by 10 sensors

As can be seen in Figures 18 and 19, small changes were made to the results compared to the previous cases, which still improved the energy consumption and transmitted packet in the networks with fewer sensors. Only the distance between fuzzification and defuzzification modes had a slight decrease, because the number of sensors in the environment and, consequently, the number of sensors in the regions decreased. According to the presented methodology in the proposed algorithm, a smaller percentage of data was reduced or removed due to the decrease in the similarity percent, since the number of sensors in the regions decreased. By comparing the fuzzification and defuzzification modes, it was concluded that decrease in the number of sensors had no significant impact on the reduction of energy consumption and increase in the network's useful lifetime, but still improved the results.

\section{CONCLUSION AND FUTURE WORK}

Sensor networks are the standard technology in wireless communications. There are hundreds and thousands of sensors in these networks and each sensor can collect data from the environment, perform computations on the collected data, and transmit the processed data to other sensors or the control center. One method to reduce the energy consumption and, as a result, increase the network lifetime is the integration of data collected from sensors covering the considered environment before the transmission in wireless sensor networks. Due to the wide range of sensor distribution, a massive amount of data is produced. Therefore, it is important to produce 
effective data. In this thesis, a new algorithm was proposed based on the methodology of fuzzy systems for the data fusion in wireless sensor networks in order to reduce energy consumption and, as a result, increase the network lifetime. The results verified the efficiency of the proposed algorithm. In the future works, the proposed method can be used in different protocols and different scenarios. One of the things that can be done in this regard is to consider the number of clusters as a variable (which was divided into 9 constant clusters in the proposed algorithm). Using learning algorithms can also help obtain better fuzzy rules.

\section{REFERENCES}

[1] Akyildiz, I. F. (2010). Wireless sensor networks. John Wiley \& Sons

[2] Abdelgawad. A, B. M. (2012). Resource-Aware data fusion algorithms for wireless sensor networks. Springer Science \& Business Media.

[3] Allahverdi, N. (2002). An application of artificial intelligence. Expert systems.

[4] Sargolzaei. Javad, K. M. (2008). Fuzzy inference system to modeling of crossflow milk ultrafiltration. Applied Soft Computing, 8(1), 456-465.

[5] Brooks. Richard R, I. S. (1998). Multi-sensor fusion: fundamentals and applications with software. PrenticeHall, Inc.

[6] E.F. Nakamura, A. A. (2007). Information Fusion for Wireless Sensor Networks. ACM Computing Surveys, 39.

[7] Yager. R R, Z. L. (2012). An introduction to fuzzy logic applications in intelligent systems. Springer Science \& Business Media.

[8] Boulis. Athanassios, G. S. (2003). Aggregation in sensor networks: an energy-accuracy trade-off. Ad hoc networks, 1(2), 317-331.
[9] Boyd. Stephen, G. A. (2005). Gossip algorithms: Design, analysis and applications. INFOCOM 2005. 24th Annual Joint Conference of the IEEE Computer and Communications Societies. Proceedings IEEE. 3, pp. 1653-1664. IEEE.

[10] Chen. Jen-Yeu, ,. P. (2006). Robust computation of aggregates in wireless sensor networks: distributed randomized algorithms and analysis. Parallel and Distributed Systems, IEEE Transactions on, 17(9), 9871000.

[11] Polastre. Joseph, H. J. (2004). Versatile low power media access for wireless sensor networks. Proceedings of the 2nd international conference on Embedded networked sensor systems (pp. 95-107). ACM.

[12] Huang. Pei, X. L. (2013). The evolution of MAC protocols in wireless sensor networks: A survey. Communications Surveys \& Tutorials, IEEE, 15(1), 101120.

[13] Kim. Jong-Myoung, P. S.-H.-J.-M. (2008). CHEF: cluster head election mechanism using fuzzy logic in wireless sensor networks. Advanced communication technology, 2008. ICACT 2008. 10th international conference on. 1, pp. 654-659. IEEE.

[14] Fathi, M., Maihami, V., \& Moradi, P. (2013). Reinforcement Learning for Multiple Access Control in Wireless Sensor Networks: Review, Model, and Open Issues. Wireless personal communications, 72(1), 535547.

[15] Fathi, M.; Maihami, V. (2015), "Operational State Scheduling of Relay Nodes in Two-Tiered Wireless Sensor Networks," in Systems Journal, IEEE, vol.9, no.3,pp.686-693. 